\title{
SYSTEMATIC CHECKLIST WITH A REVIEW OF PUBLICATIONS OF FAMILY HALICTIDAE OF EGYPT (ORDER: HYMENOPTERA) 1- SUBFAMILY: HALICTINAE
}

\author{
EL-AKKAD, M. K. and EMADELDIN A. M. BIBARS
}

Plant Protection Research Institute, ARC, Dokki, Giza, Egypt

(Manuscript received 12 February 2015)

\begin{abstract}
A systematic checklist of the Egyptian species of family Halictidae (Order: Hymenoptera) is presented, consisting of 62 species belonging to 4 subfamilies, 12 genera and 18 subgenera. The first part of this checklist includes subfamily Halictinae, consisting of one tribe, 4 genera, 11 subgenera and 38 species. The species Sphecodes (Sphecodes) ephippius (Linné, 1767) was added to the Egyptian fauna. The checklist is arranged according to species membership in higher - level taxa, based on the most recent literatureof family Halictidae.
\end{abstract}

\section{INTRODUCTION}

Family Halictidae is one of the greatest families of all bees. It is one of the most diverse families of order Hymenoptera (Pesenko et al., 2000 \& Michener, 2007). More than 500 species of cultivated plants are entomophilous, their yield directly depends on pollination activity of insects. The members of family Halictidae are considered one of the most important and efficient pollinators of many different crops (Michener, 2007).

Family Halictidae includes four subfamilies (Halictinae, Nomioidinae, Nomiinae and Rophitinae) of about 72 genera and nearly 3500 currently recognized species (Michener, 2007). Halictid bees of subfamily Halictinae of Egypt and Palaearctic region were studied by several scholars such as: Friese (1916); Alfken (1927); Blüthgen (1933 \& 1934); Ebmer (1976 \& 1995); Warncke (1975 \& 1992); Pesenko (1984);ElAkkad (1993); Pesenko et al. (2000); El-Akkad \& kamel (2002); Michener (2007); Pauly (2012); Bogusch \& Straka (2012) and Ascher \& Pickering (2014).

The present work is carried out to clarify the recent taxonomic status of the subfamily Halictinae and its members in Egypt. 


\section{MATERIALS AND METHODS}

The checklist of family Halictidae of Egypt is based on all records found in the available entomological literature and those reviewed from the preserved specimens in the main Egyptian insect collections. These collections are: the Egyptian Entomological Society collection, Ain Shams University collection and collection of the Plant Protection Research Institute, Ministry of Agriculture. Identification of the specimens had been confirmed early by A. W. Ebmer (Austria) and the late Prof. Dr. K. Warncke (Germany), the specialists of Halictidae of Palaearctic region. These specimens preserved in the collection of Plant Protection Research Institute, Ministry of Agriculture. The nomenclature and taxonomic status are adopted from the following sources: Pesenko (1984); Warncke (1992); Ebmer (1976 \& 1995); Michener (2007), Bogusch \& Straka (2012), Pauly's Atlas Hymenoptera online (2012) and Ascher \& Pickering (2014). The checklist is arranged according to species membership in higher - level taxa, based on the most recent literature. Synonyms, taxonomic notes and published records from Egypt are included.

\section{Family: Halictidae}

\section{Subfamily: Halictinae}

Tribe: Halictini

\section{Genus: Halictus Latreille,1804}

The genus Halictus Latreille is mostly a Palaearctic group in its occurrence and includes 12 subgenera and 90 currently species (Pesenko, 1984). Only 3 species belonging to 3 subgenera are recorded from Egypt as follows:

Subgenus: Halictus Latreille, 1804, Nouveau Dictionnaire d'Histoire Natur. , Vol.

24:182

\section{1- Halictus (Halictus) brunnescens (Eversmann, 1852)}

=Hylaeus brunnescens Eversmann, 1852, Bull. Soc. Moscow, 25: 36, ․

= Halictus brunnescens Smith, 1854, Catalogue of Hymen. Part (2): 423, to.

\section{Published records from Egypt:}

Friese (1916): 29-30: Halictus 4- cinctus var. aegyptiacus var. n. \&Halictus 4- cinctus var. rubripes var. n. Blüthgen (1925), Konowia, 2 (1-2): 69: (key to females), 81 (key to males): Halictus quadricinctus var. aegyptiacus Friese. Blüthgen (1933): 16: Halictus quadricinctus var. aegyptiaca Friese \& Halictus quadricinctus subsp. rufipes (F.). Blüthgen (1934): 188: Halictus quadricinctus var. aegyptiaca Friese. El-zoheiry \& Mohamed (1949): 75: Halictus quadricinctus var. aegyptiacus Friese. Shalaby (1958):

53: Halictus quadricinctus F. \& Halictus quadricinctus var. aegyptiacus Friese. Ebmer (1976), Acta Musei Moravia, 59:186-187 (Distribution): Halictus (Halictus) 
brunnescens (Eversmann). Warncke (1975): 112: Halictus (Halictus) quadricinctus spp. aegyptiacus Frieze. El-Akkad (1993): 30 (key to females), 31 (key to males), 33-

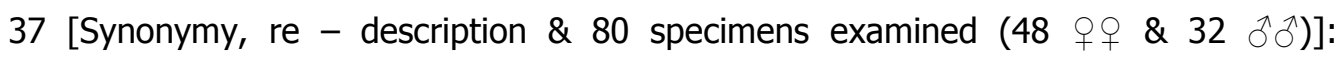
Halictus (Halictus) brunnescens (Eversmann). Dikmen \& Aytekin (2011), Turkey Journal Zoology, 35 (4): 539 - 540 (Distribution): Halictus (Halictus) brunnescens (Eversmann). Dikmen et al. (2011), Zoology in the Middle East, 54: 84 (Synonym and distribution): Halictus (Halictus) brunnescens (Eversmann). Saini \& Vikram (2012): 151 (Distribution): Halictus (Halictus) brunnescens (Eversmann). Pauly (2012): Halictus (Halictus) brunnescens (Eversmann).

\section{Subgenus: Hexataenites Pesenko, 1984: 348}

\section{2- Halictus (Hexataenites) resurgens Nurse, 1903}

= Halictus resurgens Nurse, 1903, Ann. Magazine of Nat. Hist.: 542.

= Lucasius holtzi Schulz, 1906, Spolia Hymenopterologica: 49, ふðج .

\section{Published records from Egypt:}

Blüthgen (1923), Konowia, 2 (1-2):73: (key to females), 124 (key to males): Halictus holtzi Schulz. Blüthgen (1933): 15: Halictus holtzi Schulz. Blüthgen (1934): 188: Halictus holtzi Schulz. El-zoheiry \& Mohamed (1949): 75: Halictus holtzi Schulz. Shalaby (1958): 53: Halictus holtzi Schulz. Warncke (1975): 111: Halictus (Halictus) frontalis ssp. turkomannus Pérez, = Halictus holtzi Schulz (Comb. n.). Ebmer (1976), Linzer Biologische Beitrage, 8/2: 396: Halictus (Halictus) turkomannus Pérez $=H$. holtzi Schulz = H. asiaminoris Strand. El-Akkad (1993): 30 (key to females), 32 (key to males); 40 - 43 [Synonymy, re - description \& 64 specimens examined (36 우 \&

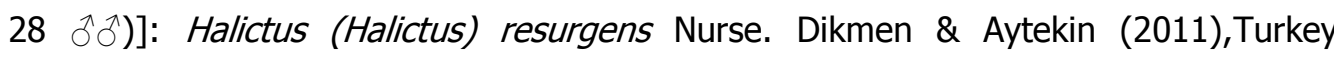
Journal Zoology, 35(4):539 - 540 (Distribution): Halictus (Hexataenites) resurgens Nurse. Dikmen et al. (2011), Zoology in the Middle East (54): 86: Halictus (Hexataenites) resurgens Nurse. Saini \& Vikram (2012): 151 (Distribution): Halictus (Hexataenites) resurgens Nurse. Pauly (2012): Halictus (Hexataenites) resurgens Nurse.

\section{Subgenus: Argalictus Pesenko, 1984: 348}

\section{3- Halictus (Argalictus) senilis (Eversmann,1852)}

= Hylaeus senilis Eversmann, 1852, Bull. Soc. Moscow, 25: 38, 위.

\section{Published records from Egypt:}

Blüthgen (1923), Konowia, 2 (1-2):75: (key to females); 80 (key to males): Halictus senilis (Eversmann); 125 (key to males): Halictus aegypticola Strand. Blüthgen (1933): 15-16: Halictus senilis Eversmann; Halictus senilis var. fucosa Mor. (ठ) \& Halictus aegypticola Strand $(\AA)$. Blüthgen (1934): 188: Halictus senilis (Eversmann). El-zoheiry \& Mohamed (1949): 75: Halictus senilis Eversmann. Shalaby (1958): 53: 
Halictus senilis Eversmann. Ebmer (1974), Acta Musei Moravia, 59: 189:(Distribution): Halictus (Halictus) senilis (Eversmann). Warncke (1975): 109: Halictus (Halictus) senilis (Eversmann). El-Akkad (1993): 30 (key to females), 31 (key to males), 44 - 48 [Synonymy, re - description \& 155 specimens examined (90 우 \& $65 \hat{\circ} \widehat{\jmath})$ )]: Halictus (Halictus) senilis (Eversmann). Dikmen \& Aytekin (2011), Turkey Journal Zoology, 35(4):539 (Synonymy \& distribution): Halictus (Argalictus) senilis (Eversmann). Pauly (2012): Halictus (Argalictus) senilis (Eversmann).

\section{Genus: Seladonia Robertson, 1918}

Seladonia Robertson, 1918, Entomological News 29: 91.

The genus Seladonia Robertson is considered as a separate genus and was subdivided into six subgenera namely Mucoreohalictus Pesenko; Pachyceble Moure; Paraseladonia Pauly; Placidohalictus Pesenko; Seladonia Robertson and Vistitohalictus Blüthgen (Pesenko (2004), Zoosystematica Rossica, 13: 102-113). It includes 102 currently recognized species, 72 of which are Palaearctic species (Pesenko (2006), Esakia, 46: 53). Only 4 species belonging to 3 subgenera are represented in Egypt as follows:

Subgenus: Mucoreohalictus Pesenko, 2004, Zoosystematica Rossica, 13:102 4- Seladonia (Mucoreohalictus) polinosus thevestensis (Pérez, 1903).

=Halictus pollinosus Sichel, 1860, Ann. Soc. Entom. France, 8: 763, ㅇ. = Halictus carinaeventris Morawitz, 1876, Turkestan Mellifera: 226, $\widehat{0}$.

\section{Published records from Egypt:}

Blüthgen (1933): 17: Halictus pollinosus Sichel = Halictus carinaeventris Morawitz. Elzoheiry \& Mohamed (1949): 75: Halictus pollinosus Sichel. Shalaby (1958): 53: Halictus pollinosus Sichel. Ebmer (1975), Mitteilungen aus dem Zoologischen Museum in Berlin, 51 (2):169 - 171 (Synonymy \& distribution), 175 (key to males); 176 (key to females): Halictus (Vestitohalictus) pollinosus pollinosus Sichel. Warncke (1975): 107: Halictus (Vestitohalictus) pollinosus Sichel. El-Akkad (1993): 57 (key to females), 58 (key to males), 66 - 69 (Synonymy, re - description \& 17 specimens examined (13 우 \& $\left.4 \widehat{\partial}^{\lambda}\right)$ )): Halictus (Vestitohalictus) pollinosus Sichel. Pesenko (2006), Esakia, 46: 56 - 65: (key to males and females), 65- 66 (Distribution): Seladonia (Mucoreohalictus) pollinosa Sichel. Pauly (2012): Vestitohalictus polinosus thevestensis (Pérez). Ascher and Pickering (2014): Halictus (Mucoreohalictus) pollinosus thevestenis (Pérez); valid species.

Subgenus: Seladonia Robertson, 1918, Entomological News 29: 91.

5- Seladonia (Seladonia) Iucidipennis (Smith, 1853).

= Halictus lucidipennis Smith, 1853, Catalogue of Hymen. Part (1): 62, đo $q$. 


\section{Published records from Egypt:}

Alfken (1927): 104 - 105: Halictus dives Pérez. Blüthgen (1933): 16 - 17: Halictus varipes var. koptica var. n. $+\hat{\jmath}$. Blüthgen (1934): 188: Halictus varipes var. dives Pérez. El-zoheiry \& Mohamed (1949): 75: Halictus varipes var. koptica Blüthgen \& Halictus varipes var. dives Pérez. Shalaby (1958): 53 - 54: Halictus dives Pérez; Halictus varipes var. dives Pérez and Halictus varipes var. koptica Blüthgen. Ebmer (1974), Acta Musei Moravia, 59: 191: Halictus (Seladonia) varipes Mor. El-Akkad (1993): 50 - 55 (Synonymy, re - description \& 442 specimens examined (377 우우 \&

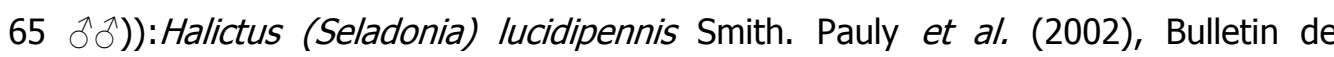
I'Institut Royal des Sciences Naturelles de Belgique, Entomologie, 72: 202 (Synonymy \& distribution): Halictus (Seladonia) lucidipennes Smith. Dawut \& Tadauchi (2003): Esakia, (43): 108 - 111 (Re- description of female; distribution and floral association in Bangladesh): Halictus (Seladonia) varipes Mor. Pesenko (2006): Esakia, 46: 61 (key to females), 64 (key to males), 73 - 74 (Synonymy \& distribution): Seladonia (Seladonia) lucidipennes (Smith). Pauly (2008), Zoologische Mededelingen, Leiden, 82: 393-394 (Synonymy): Seladonia (Seladonia) lucidipennes (Smith). Saini \& Vikram (2012): 152 (Distribution): Seladonia (Seladonia) lucidipennes (Smith).

\section{Subgenus: Vestitohalictus Blüthgen, 1961, Beiträge zur Naturkundlichen,}

19: 287.

= Mucoreohalictus Pesenko, 2004, Zoosystematica Rossica, 13: 102 (Michener, 2007: 370; new synonymy)

\section{6- Seladonia (Vestitohalictus) cupidus (Vachal, 1902).}

= Halictus cupidus Vachal, 1902, Rev. Russ. Entomology (2): 230, ô.

\section{Published records from Egypt:}

Ebmer (1976):231: Synonymy and distribution (Egypt: Fayed; Fayoum; Wadi hoff; Wadi Digla; Sinai [Wadi Feiran and Al Tor] and Israel): Halictus (Vestitohalictus) cupidus Vachal. El-Akkad (1993): 57 (key to females), 58 (key to males), 59-60

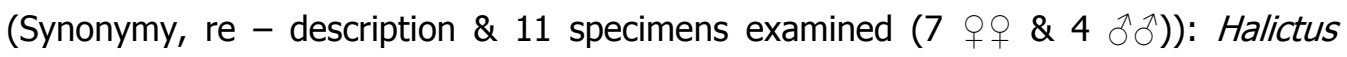
(Vestitohalictus) cupidus Vachal. Pauly (2012): Vestitohalictus cupidus (Vachal).

\section{7- Seladonia (Vestitohalictus) pici (Pérez, 1895).}

= Halictus pici Pérez, 1895, Espéces nouvelles de Melliféres de Barbariae: 53, ㅇ.

\section{Published records from Egypt:}

Vachal (1902): Rev. Russ. Entomology, 2: 230: Halictus extorris sp. n. $\hat{\jmath}$, Loc. Type: Egypt. Blüthgen (1933): 17: Halictus pici Péres.El-zoheiry \& Mohamed (1949): 75: Halictus pici Péres. Shalaby (1958): 53- 54: Halictus pici Péres \& Halictus vestitus Péres. Ebmer (1976):228 - 230: (comparing between H. pici and H. cupidus): Halictus (Vestitohalictus) pici Pérez. El-Akkad (1993): 57 (key to females), 58 (key to males), 


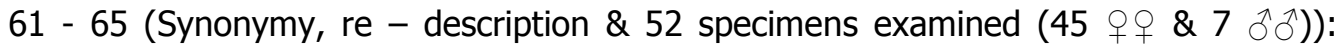
Halictus (Vestitohalictus) pici Pérez. Pauly (2012): Vestitohalictus pici (Pérez).

\section{Genus: Lasioglossum Curtis, 1833}

Lasioglossum Curtis, 1833, British Entomology, Vol. 10, pls. 434-481.

The genus Lasioglossum Curtis is divided into two series: the Lasioglossum seriesconsists of those subgenera in which the second submarginal crossvein is strong. The Hemihalictus series consists of those in which the second submarginal crossvein is weaker than the first. This genus includes 18 subgenera and nearly 1200 species worldwide (Michener, 2007). 23 species belonging to four subgenera are represented in Egypt as follows:

Subgenus: Ctenonomia Cameron, 1903, Journal of the Straits Branch of the Royal Asiatic Society, 39: 107. The subgenus Ctenonomia belongs to Lasioglossum series. It includes four species in Egypt as follows:

\section{8- Lasioglossum (Ctenonomia) fasciger (Strand, 1909)}

= Halictus fasciger Strand, 1909, Archiv für Naturgeschichte 75 (1): 32, 우․

\section{Published records from Egypt:}

Blüthgen (1933): 20: Halictus fasciger Strand. Bytinski - Salz \& Ebmer (1974):194195 (Distribution): Lasioglossum (Evylaeus) fasciger (Strand). El-Akkad (1993): 95 (key to females), 100 (key to males), 126 - 129 (Synonymy, re - description \& 16 specimens examined (10 +q \& $\left.6 \hat{\partial^{\lambda}}\right)$ ): Lasioglossum (Evylaeus) fasciger (Strand). Ascher and Pickering (2014): Lasioglossum (Ctenonomia) fasciger (Strand).

\section{9- Lasioglossum (Ctenonomia) gibber (Vachal, 1892)}

$=$ Halictus gibber Vachal, 1892, Entomology France, 61:136, o.

= Halictus gibber Blüthgen, 1925, Archiv für Naturgeschiche: 101, đ̂.

\section{Published records from Egypt:}

Blüthgen (1933): 18: Halictus gibber Vachal. El-zoheiry \& Mohamed (1949): 75: Halictus gibber Vachal. Shalaby (1958): 53: Halictus gibber Vachal. Bytinski - Salz \& Ebmer (1974): 195: Lasioglossum (Incertum) gibber (Vachal). El-Akkad (1993): 76 (key to females), 77 (key to males), 78 - 81 (Synonymy, re - description \& 54 specimens examined (36 우 \& $18 \hat{\sigma}^{\widehat{\lambda}}$ )): Lasioglossum (Ctenonomia) gibber (Vachal, 1892). Pauly (2012): Lasioglossum (Ctenonomia) gibber (Vachal). Ascher and Pickering (2014): Lasioglossum (Ctenonomia) gibber (Vachal).

\section{0- Lasioglossum (Ctenonomia) Iuridipes (Vachal, 1892)}

=Halictus Iuridipes Vachal, 1892, Entomology France, 61: 136, ․

\section{Published records from Egypt:}


Blüthgen (1934): 189: Halictus luridipes Vachal. El-zoheiry \& Mohamed (1949): 75: Halictus luridipes Vachal. Shalaby (1958): 53: Halictus Iuridipes Vachal. El-Akkad (1993): 77 (key to males and females), 82 - 85 (Synonymy, re - description \& 8

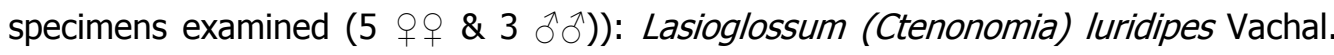
Ascher and Pickering (2014): Lasioglossum (Ctenonomia) luridipes (Vachal).

\section{1- Lasioglossum (Ctenonomia) vagans (Smith, 1857)}

=Halictus vagans Smith, 1857, Journal of the Proceeding of the Linnean Society of London, Zoology (2): 42, ㅇ.

= Halictus cattulus Vachal, 1894, Ann. Mus. Civ. Genova, 34: 437, 9 .

\section{Published records from Egypt:}

Alfken (1927): 104: Halictus cattulus Vachal. Blüthgen (1933): 18: Halictus vagans Smith. Blüthgen (1934): 189: Halictus vagans Smith. El-zoheiry \& Mohamed (1949): 75: Halictus vagans Smith. Shalaby (1958): 53: Halictuscattulus Vachal \& Halictus vagans Smith. Ebmer (1974), Acta Musei Moravia, 59:197 (Distribution): Lasioglossum (Lasioglossum) vagans Smith. Bytinski - Salz \& Ebmer (1974):181 (Distribution): Lasioglossum (Lasioglossum) vagans Smith. Sakagami (1989), Journal Kansas Entomology Soc., 62 (4):509 (key to species): Lasioglossum (Ctenonomia) vagans Smith. El-Akkad (1993): 76 - 77 (key to males and females), 86 - 93 (Synonymy, re -

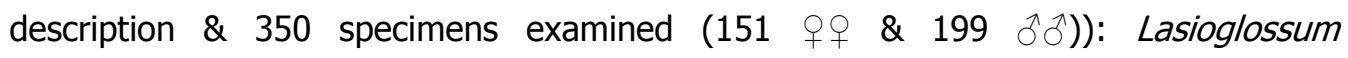
(Ctenonomia) vagans chaldoeorum (Morice). Ebmer (1998), Linzer biologische Beitrage, 30 (1):377 (Distribution): Lasioglossum (Ctenonomia) vagans (Smith). Murao et al. (2009), Esakia (49):90: Lasioglossum (Ctenonomia) vagans (Smith). Saini \& Vikram (2012): 157 (Distribution): Lasioglossum (Ctenonomia) vagans (Smith). Pauly (2012): Lasioglossum (Ctenonomia) vagans (Smith). Ascher and Pickering (2014): Lasioglossum (Ctenonomia) vagans (Smith).

Subgenus: Dialictus Robertson, 1902: Canadian Entommology, 34:248.

The members of subgenus Dialictus having metallic green reflections and the second submarginal crossvein is weaker than the first. This subgenus belongs to Hemihalictus series (Michener (2007). Three species of subgenus Dialictus are recorded from Egypt

\section{2- Lasioglossum (Dialictus) albovirens (Pérez, 1895)}

= Halictus albovirens Pérez, 1895, Espéces nouvelles de Melliféres de Barbaria: 52 -

53, ํํㅇ.

\section{Published records from Egypt:}

Blüthgen (1925), Archiv für Naturgeschichte, 90 (1924):132, §̋o : Halictus pseudoleptocephalus sp. n. Warncke (1975): 86: Halictus (Microhalictus) albovirens (Pérez). Ebmer (1976):244: Lasioglossum (Evylaeus) albovirens albovirens (Pérez) \& Lasioglossum (Evylaeus) albovirens pseudoleptocephalum (Blüthgen). El-Akkad 
(1993): 95 (key to females), 107 - 109 (Synonymy, re - description of female \& one female examined): Lasioglossum (Evylaeus) albovirens pseudoleptocephalum (Blüthgen). Pauly (2012): Lasioglossum (Dialictus) albovirens (Pérez).

\section{3- Lasioglossum (Dialictus) leptocephalum (Blüthgen, 1923)}

= Halictus leptocephalus Blüthgen, 1923, Archiv für Natturgeschichte: 245, +.

\section{Published records from Egypt:}

Blüthgen (1934): 190: Halictus leptocephalus Blüthgen. El-zoheiry \& Mohamed (1949): 75: Halictus leptocephalus Blüthgen. Shalaby (1958): 53: Halictus leptocephalus Blüthgen. Bytinski- Salz \& Ebmer (1974): 183: Lasioglossum (Evylaeus) leptocephalum (Blüthgen); 195- 197: Original description of female of Lasioglossum (Evylaeus) leptocephalumsinaiticum Ebmers sp. n o.El-Akkad (1993): 94 (key to females), 130 - 132 (Synonymy, re - description of female; one specimen examined): Lasioglossum (Evylaeus) leptocephalum (Blüthgen). Pauly (2012): Lasioglossum (Dialictus) leptocephalum (Blüthgen).

\section{4- Lasioglossum (Dialictus) mandibulare (Morawitz, 1866)}

= Halictus mandibular Morawitz, 1866, Soc. Entom. Ross. (4): 3 - 28.

= Halictus carneiventris Dours, 1872, Revue et magasin de Zool. (2) 23: 311, + .

= Halictus carneiventris Blüthgen, 1925, Archiv für Natturgeschichte: 118, 0 .

\section{Published records from Egypt:}

Blüthgen (1933): 20: Halictus carneiventris Dours. El-zoheiry \& Mohamed (1949): 75: Halictus carneiventris Dours. Shalaby (1958): 53: Halictus carneiventris Dours. Bytinski - Salz \& Ebmer (1974): 194 (Distribution): Lasioglossum (Evylaeus) carneiventre (Dours). Warncke (1975): 104: Halictus (Pyghalictus) mandibulare Morawitz = Halictus carneiventris Dours synonymnew. El-Akkad (1993): 96 (key to females), 101 (key to males), 113 - 117 (Synonymy, re - description and 51 specimens examined (40 우 \& 11 周)): Lasioglossum (Evylaeus) carneiventre (Dours). Pauly (2012): Lasioglossum (Dialictus) mandibular (Morawitz).

Subgenus: Evylaeus Robertson, 1902, Canadian Entomology, 34: 247

This subgenus is the largest subgenus of the family Halictidae. It includes 318 currently recognized species in the Palaearctic Region (Pesenko, 2007), Zootaxa, 1500: 1 - 54). This subgenus belongs to Hemihalictus series (Michener, 2007) and having the second submarginal crossvein is weaker than the first. It is represented by 14 species in Egypt

\section{5- Lasioglossum (Evylaeus) ablenum (Blüthgen, 1934)}

= Halictus ablenus Blüthgen, 1934: 198- 200, ภ̊.

\section{Published records from Egypt:}


Blüthgen (1934): 198 - 200: (Original description): Halictus ablenus sp. n. El-zoheiry \& Mohamed (1949): 75: Halictus ablenus Blüthgen. Shalaby (1958): 53: Halictus ablenus Blüthgen. El-Akkad (1993): 99 - 102 (key to males); 162 - 165 (Re description of type): Lasioglossum (Evylaeus) ablenum Blüthgen. Saini \& Vikram (2012): 154: (Distribution): Lasioglossum (Evylaeus) ablenum (Blüthgen). Pauly (2012): Lasioglossum (Evylaeus) ablenum (Blüthgen).

\section{6- Lasioglossum (Evylaeus) articulare (Pérez, 1895)}

= Halictus articularis Pérez, 1895, Espéces nouvelles Melliféres Barbaria: 54. ㅇ.

= Halictus romanettii Blüthgen, 1923, Archiv für Natturgeschichte: 276. ^ơ.

\section{Published records from Egypt:}

Blüthgen (1924), Konowia, 3:60 - 62 (key to females), 262 (key to males): Halictus romanettii Blüthgen. Blüthgen (1933): 19: Halictus articularis Pérez. Blüthgen (1934): 190: Halictus articularis Pérez = Halictus romanettii Blüthgen. El-zoheiry \& Mohamed (1949): 75: Halictus articularis Pérez. Shalaby (1958): 53: Halictus articularis Pérez. Bytiniski-Salz \& Ebmer (1974): 185: Lasioglossum (Evylaeus) articulare (Pérez). Ebmer (1976): 249: Lasioglossum (Evylaeus) articulare (Pérez). El-Akkad (1993): 99 (key to males), 110 - 112 (Synonymy, re - description of male; 3 ô specimens examined): Lasioglossum (Evylaeus) articulare (Pérez). Pauly (2012): Lasioglossum (Evylaeus) articulare (Pérez).

\section{7- Lasioglossum (Evylaeus) clypeiferellum (Strand, 1909)}

= Halictus clypeiferellus Strand, 1909, Archiv für Naturgesschichte 75: 28, ㅇ.

= Halictus testaceohirtulus Blüthgen, 1929, Konowia, 8:65, ô.

\section{Published records from Egypt:}

Blüthgen (1929), Konowia, 8: 65-67 (Original description): Halictus testaceohirtulus $\hat{0}$, sp. n.Blüthgen(1933): 19: Halictus clypeiferellus Strand.Bytiniski - Salz \& Ebmer (1974): 188: Lasioglossum (Evylaeus) clypeiferellum (Strand). Warncke (1975): 92: Halictus (Evylaeus) clypeiferellus Strand. El-Akkad (1993): 101 (key to males), 118 - 120 (Synonymy, re - description of male; one male examined): Lasioglossum (Evylaeus) clypeiferellum (Strand). Pauly (2012): Lasioglossum (Evylaeus) clypeiferellum (Strand).

\section{8- Lasioglossum (Evylaeus) decolor (Pérez, 1895)}

= Halcitus decolor Pérez, 1895, Espéces nouvelles de Melliféres Barbaria: 54, + .

= Halcitus decolor Blüthgen, 1934: 192- 194, §̂.

\section{Published records from Egypt:}

Blüthgen (1934): 190 - 194, Original description of Halictus decolor sp. n. $\hat{\jmath}$. Elzoheiry \& Mohamed (1949): 75: Halictus decolor Pérez. Shalaby (1958): 53: Halictus decolor Pérez. El-Akkad (1993): 99 (key to males), 121- 122 (Re - description of 
male; ô paratype examined): Lasioglossum (Evylaeus) decolor (Pérez). Ebmer (1995): 556: (Distribution: Algeria, Tunisia, Lybia and Egypt): Lasioglossum (Evylaeus) decolor (Pérez). Pauly (2012): Lasioglossum (Evylaeus) decolor (Pérez).

\section{9- Lasioglossum (Evylaeus) elbanum (Blüthgen, 1934)}

= Halictus elbanus Blüthgen, 1934: $194-196$, ồ .

\section{Published records from Egypt:}

Blüthgen (1934): 190 \& 192 - 194: Original description of male and female of Halictus elbanus sp. n. El-zoheiry \& Mohamed (1949): 75: Halictus elbanus Blüthgen. Shalaby (1958): 53: Halictus elbanus Blüthgen. Bytiniski-Salz \& Ebmer (1974): 185: Lasioglossum (Evylaeus) elbanum Blüthgen. Ebmer (1975): Linzer Biologische Beitrage, 7 (1):103 (Distribution): Lasioglossum (Evylaeus) elbanum (Blüthgen). ElAkkad (1993): 96 (key to females), 100 (key to males) 122- 125 (Re - description; female paratype examined from Gabal Elba, 26/1/33): Lasioglossum (Evylaeus) elbanum (Blüthgen). Ebmer (1995): 555: (Distribution: Sudan, Egypt and Israel): Lasioglossum (Evylaeus) elbanum (Blüthgen) Pauly (2012): Lasioglossum (Evylaeus) elbanum (Blüthgen).

\section{0- Lasioglossum (Evylaeus) leptorhynchum (Blüthgen, 1931)}

= Halictus leptorhynchus Blüthgen, 1931, Mitt. Zool. Mus. Berlin, 17: 360 - 362, Holotype: 1 q Wadi Um Elek (15/10/19); Paratype: 1 q Wadi Hussein (31/ 5/19), Egypt.

\section{Published records from Egypt:}

Blüthgen (1933): 19: Halictus leptorhynchus Blüthgen. Bytinski - Salz \& Ebmer (1974): 193 Lasioglossum (Evylaeus) leptorhynchum (Blüthgen). El-Akkad (1993): 94 (key to females), 133 - 134 ( $\mathrm{Re}$ - description of female; Holotype and paratype examined): Lasioglossum (Evylaeus) leptorhynchum (Blüthgen). Pauly (2012): (Distribution: Egypt): Lasioglossum (Evylaeus) leptorhynchum (Blüthgen).

\section{1- Lasioglossum (Evylaeus) malachurum (Kirby, 1802)}

= Melitta malachura Kirby, 1802, Monographia Apum Angliae, Vol. II: 67. ๆ.

= Hylaeus apicalis Schenck, 1853, Jahr. Ver. Naturk. Nassau, 9 (1): 161. đ̂.

\section{Published records from Egypt:}

Blüthgen (1924): Konowia, 3: 81 \& 90 (key to females); 269 (key to males): Halictus malachurus Kirby. Blüthgen (1933): 19: Halictus malachurus (Kirby) = H. longulus Smith. Bytiniski-Salz \& Ebmer (1974): 186: Lasioglossum (Evylaeus) malachurum (Kirby). Warncke (1975): 100: Halictus (Calchalictus) malachurus (Kirby). Ebmer (1976): 248: Lasioglossum (Evylaeus) malachurum (Kirby) \& Lasioglossum (Evylaeus) malachurum sharificus Cockerell is a synonym new. Ebmer (1976), Linzer Biologische Beitrage, 8/2:400: Lasioglossum (Evylaeus) malachurum (Kirby). El-Akkad (1993): 90 
(key to females); 96 (key to males); 130 - 133 (Synonymy \& re - description; 2 specimens examined): Lasioglossum (Evylaeus) malachurum (Kirby). Pauly (2012): (Distribution: Western Palaearctic, North Africa from the Canary Islands, Morocco, Tunisia, to Egypt; Middle East, very common in Turkey, rare in Syria, Israel and Jordon): Lasioglossum (Evylaeus) malachurum (Kirby).

\section{2- Lasioglossum (Evylaeus) masculum (Pérez, 1895)}

= Halictus masculus Pérez, 1895, Espéces nouvelle de Melliféres Barbaria: 55, .

\section{Published records from Egypt:}

Blüthgen (1924), Konowia, 3:58 (key to females); 262 (key to males): Halictus leucopymatus Dalla Torre \& Halictus leucopymatus var. numidus Blüthgen. BytiniskiSalz \& Ebmer (1974): 187 (Distribution) \& 201: Original description of male of Lasioglossum (Evylaeus) masculum (Pérez). Ebmer (1976): 250: Lasioglossum (Evylaeus) masculum (Pérez). El-Akkad (1993): 91 (key to females); 96 (key to males); 137 - 140 (Re - description; 10 specimens examined): Lasioglossum (Evylaeus) masculum (Pérez). Pauly (2012): (Distribution: West Palaearctic, Morocco, Algeria, Egypt, Israel, Jordon and Syria): Lasioglossum (Evylaeus) masculum (Pérez).

23- Lasioglossum (Evylaeus) mariuticum (Blüthgen, 1934)

= Halictus mariuticus Blüthgen, 1934: 196- 197, $q$.

\section{Published records from Egypt:}

Blüthgen (1934): 196 - 197 Original description of Halictus mariuticus sp. n. Elzoheiry \& Mohamed (1949): 75: Halictus mariuticus Blüthgen. Shalaby (1958): 53: Halictus mariuticus Blüthgen. Bytinski -- Salz \& Ebmer (1974): 192 Lasioglossum (Evylaeus) mariuticum (Blüthgen). El-Akkad (1993): 95 (key to females), 139 - 141 (Re - description of female; Holotype examined): Lasioglossum (Evylaeus) mariuticum (Blüthgen). Pauly (2012): (Distribution: Egypt) Lasioglossum (Evylaeus) mariuticum (Blüthgen).

\section{4- Lasioglossum (Evylaeus) mesosclerum (Pérez, 1903)}

= Halictus mesosclerus Pérez, 1903, Espéces nouvelles de Melliféres (58): 43, o.

\section{Published records from Egypt:}

Blüthgen (1933): 19: Halictus mesosclerus Pérez. Blüthgen (1934): 190: Halictus mesosclerus Pérez. El-zoheiry \& Mohamed (1949): 75: Halictus mesosclerus Pérez. Shalaby (1958): 53: Halictus mesoclerus Pérez. Ebmer (1974), Acta Musei Moravia, 59: 202: Lasioglossum (Evylaeus) mesosclerum (Pérez). Warncke (1975): 91: Halictus (Evylaeus) mesosclerus (Pérez). Ebmer (1976), Linzer Biologische Beitrage, 8/2:404: Lasioglossum (Evylaeus) mesosclerum (Pérez). El-Akkad (1993): 98 (key to females), 102 (key to males), 146 - 149 (Synonymy, re - description and 4 specimens 
examined (3우 \& 1 §ै)): Lasioglossum (Evylaeus) mesosclerum (Pérez). Pauly (2012): (Distribution: Lybia and Egypt): Lasioglossum (Evylaeus) mesosclerum (Pérez).

\section{5- Lasioglossum (Evylaeus) minutissimum (Kirby, 1802)}

= Melitta minutissima Kirby, 1802, Monographia Apum Anglia, Vol. II: 63, §ึㅇ․

\section{Published records from Egypt:}

Blüthgen (1933): 19: Halictus minutissimus var. hollandi Saunder, 1904. Bytinski - Salz \& Ebmer (1974): 193 Lasioglossum (Evylaeus) minutissimum (Kirby). Warncke (1975): 86: Halicttus (Microhalictus) minutissimus (Kirby). El-Akkad(1993): 97 (key to females), 103 (key to males); 150- 153 (Synonymy, re - description and 13

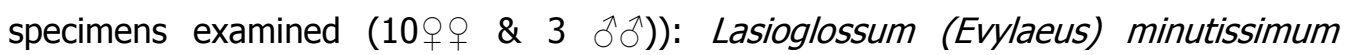
(Kirby). Pauly (2012): Lasioglossum (Evylaeus) minutissimum (Kirby).

\section{6- Lasioglossum (Evylaeus) nabardicum (Blüthgen, 1931)}

= Halictus nabardicus Blüthgen, 1931, Mitt. Zool. Mus. Berlin, 17: 367 - 368, q; Type locality: Nubian Desert.

\section{Published records from Egypt:}

Blüthgen (1933): 19: Halictus nabardicus Blüthgen. Bytinski -- Salz \& Ebmer (1974):193: new description of male of Lasioglossum (Evylaeus) nabardicum (Blüthgen); Sinai: Wadi Herban. El-Akkad (1993): 97 (key to females), 99 (key to males); 154 - 157 (Re - description): Lasioglossum (Evylaeus) nabardicum (Blüthgen). Pauly (2012): (Distribution: Sudan [Nobian desert], Sinai, Israel): Lasioglossum (Evylaeus) nabardicum (Blüthgen).

\section{7- Lasioglossum (Evylaeus) transitorium (Schenck, 1869)}

= Halictus transitorius Schenck, 1869, Jahr. Nassau. Für Naturk., 21/22:309, ㅇ.

\section{Published records from Egypt:}

Ebmer (1971): 116: Lasioglossum (Evylaeus) transitorium planulum (Pérez). Bytinski - Salz \& Ebmer (1974):191: this species occurs in a separate subspecies namely Lasioglossum (Evylaeus) transitorium planulum (Pérez)] in the Western Mediterranean. El-Akkad (1993): 97 (key to females), 102 (key to males); 158 - 161 (Synonymy, re - description): Lasioglossum (Evylaeus) transitorium planulum (Pérez). Pauly (2012) mentioned that there are three subspecies belonging to this species namely: L. (Evylaeus) transitorium transitorium (Schenck, 1869); L. (Evylaeus) transitoriumplanulum (Pérez, 1903) and L. (Evylaeus) transitorium uncinum (Vachal); the last subspecies represented in eastern Mediterranean (Cyprus, Southern Asia Minor, Syria, Israel, Jordan and Egypt).

\section{8- Lasioglossum (Evylaeus) villosulum (Kirby, 1802)}

= Melitta villosula Kirby, 1802, Monographia Apum Anglia, Vol. II: 62. $\delta$.

= Melitta punctulata Kirby, 1802, Monographia Apum Anglia, Vol. II: 62.. 


\section{Published records from Egypt:}

Blüthgen (1933): 19 Halictus villosulus (Kirby). Blüthgen (1934): 190: Halictus villosulus (Kirby). El-zoheiry \& Mohamed (1949): 75: Halictus villasulus K. Shalaby (1958): 54: Halictus villasulus W. K. Bytinski -- Salz \& Ebmer (1974): 188 - 189: Lasioglossum (Evylaeus) villosulum (Kirby). Warncke (1975): 91: Halicttus (Evylaeus) villosulus (Kirby). Ebmer (1976): 253: Lasioglossum (Evylaeus) villosulum (Kirby). Ebmer (1976), Linzer Biologische Beitrage, 8/2: 402: Lasioglossum (Evylaeus) villosulum (Kirby) belonging to villosolum group. El-Akkad (1993): 98 (key to females), 102 (key to males); 162 - 165 (Synonymy, re - description; specimens examined 9 우)): [Lasioglossum (Evylaeus) villosulum (kirby)]. Ebmer (2008), Linzer Biologische Beitrage, 40 (1): 549 - 579: Original description of Lasioglossum (Evylaeus) villosulum arabicum Ebmer. Saini \& Vikram (2012): 156 (Distribution): Lasioglossum (Evylaeus) villosulum (Kirby). Pauly (2012): (Synonymy \& distribution): Lasioglossum (Evylaeus) villosulum (Kirby).

\section{Subgenus: Lasioglossum Curtis, 1833, British Entomology, PI. 448.}

This subgenus belongs to Lasioglossum series, members of it have the second submarginal crossvein is strong, like the first and unlike the third one. Two species only are represented from Egypt as follows:

\section{9- Lasioglossum (Lasioglossum) aegyptiellum (Strand, 1909)}

= Halictus morbillosus var. aegyptiellus Strand, 1909, Arch. Naturg., 75: 11, .

\section{Published records from Egypt:}

Blüthgen (1933): 17: Halictus aegyptiellus Cockerell. Ebmer (1970), Naturkundliches Jahrbuch der Stadt Linz: 20 (key to females); 25 (key to males); 31-33 (Synonymy \& re - description): Lasioglossum (Lasioglossum) aegyptiellum (Strand). Bytiniski- Salz \& Ebmer (1974): 179 (Distribution): Lasioglossum (Lasioglossum) aegyptiellum (Strand). Warncke (1975): 108: Halictus (Halictus) aegypticola Strand. Ebmer (1976): 235: Lasioglossum (Lasioglossum) aegyptiellum (Strand). Ebmer (1976), Linzer Biologische Beitrage, 8/2:400 Lasioglossum (Lasioglossum) aegyptiellum (Strand). El-Akkad (1993): 161 (key to females and males); 162 - 164 (Synonymy \& re - description; 3 specimens examined): Lasioglossum (Lasioglossum) aegyptiellum (Strand). Pauly (2012): Lasioglossum (Lasioglossum) aegyptiellum (Strand).

\section{0- Lasioglossum (Lasioglossum) callizonium (Pérez, 1895)}

= Halictus callizonius Pérez, 1895, Espéces nouvelle Melliféres Barbaria:54, + .

= Halictus callizonius Alfken, 1914, Mém. Soc. Entom. Belgique, 22: 190, §ิ.

\section{Published records from Egypt:}

Blüthgen (1933): 17: Halictus callizonius Pérez. Blüthgen (1934): 189: Halictus callizonius Pérez. El-zoheiry \& Mohamed (1949): 75: Halictus callizonius Pérez. 
Shalaby (1958): 53: Halictus callizonius Pérez Bytiniski- Salz \& Ebmer (1974): 178 (Distribution): Lasioglossum (Lasioglossum) callizonium (Pérez). Ebmer (1976): 235: Lasioglossum (Lasioglossum) callizonium (Pérez). El-Akkad (1993): 161 (key to females and males); 165- 170 (Re - description; 132 specimens examined): Lasioglossum (Lasioglossum) callizonium (Pérez). Pauly (2012): Lasioglossum (Lasioglossum) callizonium (Pérez).

\section{Genus: Sphecodes Latreille, 1804}

Sphecodes Latreille, 1804, Nouveau Dictionnaire d'Histoire Naturelle, Vol. 24: 129 200.

The members of the genus Sphecodes are usually black with a partly or wholly red metasoma, but sometimes, especially in males, the metasoma entirely black. The genus is widespread on all continents, except Australia. There may be 100 named Palaearctic species (Michener, 2007). El- Akkad \& Kamel (2002) illustrated and keyed seven species of Sphecodes from Egypt. Warncke (1992) added the species Sphecodes ephippius Linné to the Egyptian fauna.

\section{Subgenus: Sphecodes Latreille, 1804.}

\section{1- Sphecodes (Sphecodes) albilabris (Fabricius, 1793).}

= Nomada albilabris Fabricius, 1793, Entomologia Systematica, Vol 2: 349

\section{Published records from Egypt:}

Blüthgen (1933): 22: Sphecodes fuscipennis subsp. rubripes Spinola. Blüthgen (1934): 190:Sphecodes rubripes Spinola. Shalaby (1958): 112: Sphecodes fuscipennis Germ. Warncke (1992): 11 (key to females); 13 (key to males); 30 - 31(Synonym \& distribution): Sphecodes fuscipennis subsp. rubripes Spinola. El-Akkad \& Kamel (2002): 1583 - 1584 (key to males and females); 1584 - 1585 (Synonym, redescription \& 7 specimens examined $\left(5\right.$ 우 $\left.+2 \delta^{\lambda} \delta^{\top}\right)$ ): Sphecodes (Sphecodes) albilabris subsp. rubripes Spinola. Bogusch \& Straka (2012): 3: (key to females); 6 (key to males); 7 -8 (Synonym, distribution, Biology and taxonomic notes); 19 (Hosts): Sphecodes (Sphecodes) albilabris (Fabricius).

\section{2- Sphecodes (Sphecodes) alternates Smith, 1853}

=Sphecodes alternatus Smith, 1853, Catalogue Hymen. British Mus. I: 36, n.9.

\section{Published records from Egypt:}

Blüthgen (1933): 22: Sphecodes alternatus Smith. Blüthgen (1934): 191: Sphecodes alternatus Smith. El-zoheiry \& Mohamed (1949): 75: Sphecodes alternatus Smith. Shalaby (1958): 111: Sphecodes alternatus Smith. Warncke (1992): 11 (key to females); 14 (key to males); 26 (Synonym \& distribution): Sphecodes alternatus algeriensis Alfken- Stat. n. El-Akkad \& Kamel (2002): 1583 - 1584 (key to males and females); $1585-1586$ (Re-description\& 16 specimens examined $(9$ 우우 $+7 \hat{\delta} \widehat{\jmath})$ ): 
Sphecodes (Sphecodes) alternatus Smith. Bogusch \& Straka (2012): 4: (key to females); 6 (key to males); 8 (Synonymy; distribution \& biology); 19 (Hosts): Sphecodes (Sphecodes) alternatus Smith.

\section{3- Sphecodes(Sphecodes) ephippius (Linné, 1767)}

= Sphex ephippia Linné, 1767, Systema Naturae, Editio 12: 944.

\section{Published records from Egypt:}

Warncke (1992): 11 (key to females); 15 (key to males); 20 (Synonymy \& distribution: North Africa, from Morocco to Egypt): Sphecodes ephippius Linné. Bogusch \& Straka (2012): 4: (key to females); 6 (key to males); 10 (Synonymy, Distribution \& Biology); 20 (Hosts): Sphecodes (Sphecodes) ephippius Linné.

Note: This species is not represented by any specimens in the Egyptian Insect Collections.

\section{4-Sphecodes (Sphecodes) gibbus (Linné, 1758)}

= Sphex gibba Linnaeus, 1758, Systema Naturae, Editio 1: 571.

=Sphecodes gibbus Latreille, 1809, Genera Crustaceorum Insectorum, 4: 153.

\section{Published records from Egypt:}

Blüthgen (1933): 22: Sphecodes gibbus L. Blüthgen (1934): 191: Sphecodes gibbus L. El-zoheiry \& Mohamed (1949): 75: Sphecodes gibbus L. Shalaby (1958): 112: Sphecodes gibbus L. Warncke (1992): 11 (key to females); 13 (key to males); 29 - 30 (Synonymy \& distribution: North Africa from Morocco to Israel): Sphecodes gibbus rufispinosus Meyer (Stat. n.). El-Akkad \& Kamel (2002): 1583 - 1584: (key to males

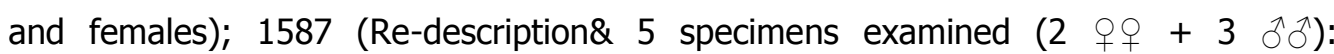
Sphecodes (Sphecodes) gibbus L. Bogusch \& Straka (2012): 4: (key to females); 6 (key to males); 11 (Synonymy, diststribution \& biology); 20 (Hosts): Sphecodes (Sphecodes) gibbus L. Saini \& Vikram (2012): 162: (Distribution): Sphecodes (Sphecodes) gibbus L.

\section{5-Sphecodes (Sphecodes) hirtellus Blüthgen, 1923}

= Sphecodes hirtellus Blüthgen, 1923, Deutsh. Entom. Zeitschr.: 502: đo \& .

\section{Published records from Egypt:}

Blüthgen (1934): 191: Sphecodes hirtellus Blüthgen: new recorded from Egypt. El-zoheiry \& Mohamed (1949): 75: Sphecodes hirtellus Blüthgen. Shalaby (1958): 112: Sphecodes hirtellus Blüthgen. Warncke (1992): 9 (key to females); 15 (key to males); 18 (Distribution: Madrid (Spain), Tanger (Morocco), Algeria and Egypt): Sphecodes hirtellus Blüthgen. El-Akkad \& Kamel (2002): 1583: (key to males and females); 1588 (one female re-described and examined): Sphecodes (Sphecodes) hirtellus Blüthgen. 


\section{6- Sphecodes (Sphecodes) olivieri Lepeletier, 1825}

= Sphecodes olivieri Lepeletier, 1825, Encycl. method. Insect, X: 448 n. 2.

= Sphecodes verticalis Hagens, 1882, Deutsh. Entom. Zeitschr 26: 219.

\section{Published records from Egypt:}

Alfken (1927): 105: Sphecodes verticalis Hag. Blüthgen (1933): 22: Sphecodes olivieri Lepeletier. Blüthgen (1934): 190: Sphecodes olivieri Lepeletier. El-zoheiry \& Mohamed (1949): 75: Sphecodes olivieri; Sphecodes olivieri var. verticalis \& Sphecodes verticalis Hag. Shalaby (1958): 112: Sphecodes olivieri \& Sphecodes verticalis Hagens. Warncke (1992): 10 (key to females); 13 (key to males); 24 (Synonymy and distribution: North Africa from Morocco to Egypt, Israel and Kuakas): Sphecodes olivieri Lep. El-Akkad \& Kamel (2002): 1583 - 1584: (key to males and females); 1588 - 1589 (Re-description \& 128 specimens examined $(60$ 우우 +68 수 $)$ : Sphecodes (Sphecodes) olivieri Lep. Bogusch \& Straka (2012): 3: (key to females); 6 (key to males); 14 - 15 (Synonymy, distribution \& biology); 21 (Hosts): Sphecodes (Sphecodes) olivieri Lep. Saini \& Vikram (2012): 163: (Distribution): Sphecodes olivieri Lep.

\section{7 - Sphecodes (Sphecodes) puncticeps Thomson, 1870}

= Sphecodes puncticeps Thomson, 1870, Opusc. Entom.: 2 \& 99, n. 6 .

\section{Published records from Egypt:}

Blüthgen (1934): 191: Sphecodes puncticeps Thomson; recorded from Egypt for the first time. Warncke (1992): 9 (key to females); 15 (key to males); 18 - 19 (Synonymy and distribution: North Africa from Morocco to Egypt and Israel): Sphecodes puncticeps Thomson. El-Akkad \& Kamel (2002): 1582 - 1583: (key to males and females); 1589 - 1590 (Re-description \& 4 males examined): Sphecodes (Sphecodes) puncticeps Thomson. Bogusch \& Straka (2012): 4: (key to females); 6 (key to males); 16 (Synonymy, distribution \& biology); 22 (Hosts): Sphecodes (Sphecodes) puncticeps Thomson.

\section{8- Sphecodes (Sphecodes) ruficrus (Erichson, 1835)}

= Dichroa ruficrus Erichson, 1835, Waltl: Reise d. Tirol etc. P: 2 \& 101.

= Sphecodes hispanicus Wesmael, 1835, Bull. Acad. Sc. Belgique 2: 286.

\section{Published records from Egypt:}

Blüthgen (1933): 22: Sphecodes ruficrus Erichson. Blüthgen (1934): 191: Sphecodes ruficrus Erichson. El-zoheiry \&Mohamed (1949): 75: Sphecodes ruficrus Erichson. Shalaby (1958): 112: Sphecodes ruficrus Erichson. Warncke (1992): 11 (key to females); 14 (key to males); 21 (Synonymy and distribution: North Africa from Morocco to Egypt and Israel): Sphecodes ruficrus Erichson. El-Akkad \& Kamel (2002): 1582 \& 1584: (key to males and females); 1590 - 1591 (Re-description\& 16 specimens examined $\left(7\right.$ 우 $\left.\left.+9 \widehat{\delta}^{\Uparrow}\right)\right)$ : Sphecodes (Sphecodes) ruficrus 
Erichson.Bogusch \& Straka (2012): 4: (key to females); 6 (key to males); 17 (Synonymy, distribution \& biology); 22 (Hosts): Sphecodes (Sphecodes) ruficrus Erichson.

\section{REFERENCES}

1. Alfken, J. D. 1927. Uber eine Bienenausbeute von Ägypten. Bull. Soc. Entom. Egypt, 1926: 102 - 106.

2. Ascher, J. S. \& Pickering, J. 2014. http://www.discoverlife.org/mp/20q?guide=Apoidea_species

3. Blüthgen, P. 1933. Ein Beitrage zur Kenntnis der Bienenfauna Äegyptens (Hymenoptera: Apidae: Halictidae: Halictinae). Bulletin de la Société Royale Entomologique d'Egypte, (1-3): 14-27.

4. - - 1934. Zweiter Beitrage zur Kenntnis der Halictinen fauna Äegyptens (Hymenoptera: Apidae: Halictidae: Halictinae). Bulletin de la Société Royale Entomologique d'Egypte, 18: 188-201.

5. Bogusch P. \& Straka J., 2012. Review and identification of the cuckoo bees of central Europe (Hymenoptera: Halictidae: Sphecodes). Zootaxa, 3311: 1- 41.

6. Bytinski - Salz \& Ebmer, A. W. 1974. The Halictidae of Israel (Hymenoptera: Apoidea). II. Genus Lasioglossum. Israel Journal of Entom., 9: $175-217$.

7. Ebmer, A.W.1976:Halictus und Lasioglossum aus Marrokko. Linzer Biologische Beitrage, 8: $205-266$.

8. --_.-1995. Asiatsche Halicidae, 3. Die Artengruppe der Lasioglossum carinate Evylaeus (Hymenoptera: Halictidae). Linzer Biologische Beitrage, 27/2: $525-652$.

9. El-Akkad, M. K. 1993. Taxonomical and ecological studies of wild bees of subfamily Halictinae (Order Hymenoptera, family Halictidae) in Egypt. Unpublished Ph. D. Thesis, faculty of Agriculture, Moshtohor, Zagazig University, $225 \mathrm{pp}$

10. El-Akkad, M. K. and Kamel, S. M. 2002. Revision of the genus Sphecodes Latreille of Egypt (Hymenoptera: Halictidae). Eygptian J. Agric. Res., 80 (4): 1579 - 1595.

11. El-zoheiry, M. S. \& Mohamed N. 1949. List of Egyptian insects in the collection of the Entomological Section, Min. of Agriculture, Egypt, 87 pp.

12. Friese, H., 1916. Die Formen des Halictus quadricinctus Fabricius, Sowie einige neue Halictus - Arten der Palaearkischen Region (Hymenoptera). Deutsche, Entom. Zeit.: $28-30$. 
13. Michener, C. D. 2007. The Bees of the World (Second Edition), Baltimore: Johns Hopkins University Press, 953 pp.

14. Pauly, A. 2012.

http://zoologie.umh.ac.be/hymenoptera/page.asp?id=67(Seladonia);id=70

(Halictus);id=89 (Ctenonomia);id=95 (Evylaeus); id=97 (Vestitohalictus); id=105 (Lasioglossum); id=128 (Dialictus).

15. Pesenko, Yu. A. 1984. A subgeneric classification of bees of the genus Halictus Latreille sensu stricto (Hymenoptera: Halictidae). Entomologicheskoe Obozrenie (Leningrad) 63: 340 - 357. [In Russian; English translation in Entomological Review, 63(3): 1-20].

16. Pesenko, Yu. A., J. Banaszak, V. G. Radchenko \& T. Cierzniak, 2000. Bees of the family Halictidae (excluding Sphecodes) of Poland: Taxonomy, ecology, bionomics. Bydgoszcz (Poland), 348 pp.

17. Saini, M. S. \& Vikram, R. S. 2012. A species checklist of family Halictidae (Hymenoptera: Apoidea) among with keys to subfamilies, genera \& subgenera from India. International Journal of Environmental Sciences, 3 (1): 134 - 166.

18. Shalaby, F. 1958. Alphabetical list of Egyptian Insects in the Collection of the Ministry of Agriculture. Ministry of Agric. Technical Bulletin No. 284, 135 pp.

19. Warncke K. 1975. Beitrage zur Systematik und Verbreitung der Furchenbienen in der Turkei (Apoidea: Halictus). Polskie Pismo entomologiczne, 45 (1): 81-128.

20. - ------1992. Die westpaläarktischen Arten der Bienengattung Sphecodes Latrielle Bericht der Naturforschende Gesellschaft Augsburg, 52: 9-64. 
قائمة تصنيفيه لفصيلة هاليكتيدى في مصر (رتبة غثائية الأجنحة)

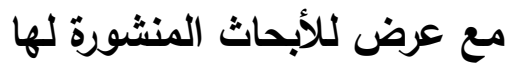

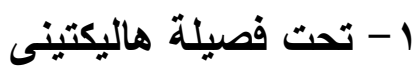

محمد كمال العقاد و عماد الدين عبد الفتاح معوض بييرس

معهد بحوث وقاية النباتات ، مركز البحوث الزراعية ، الدقى ، الجيزة ، مصر

يقدم هذا البحث قائمة تصنيفيه للأنواع التابعة لفصيلة هاليكتيدى في مصر (رتبة غشائية

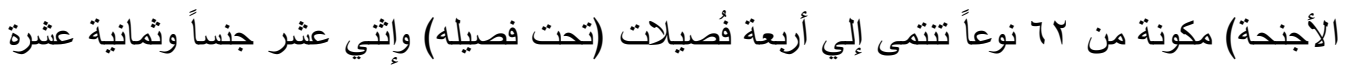
جنيساً (تحت جنس). إثنتل البحث علي قائمة للأنواع التابعة لفُصيلة (تحت فصيلة) هاليكتينى والتي تتكون من أربعة أجناس

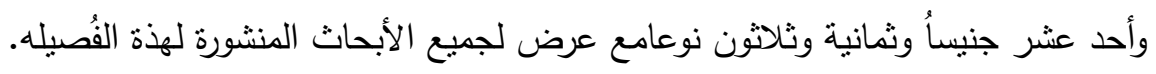

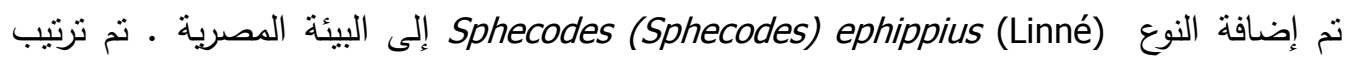

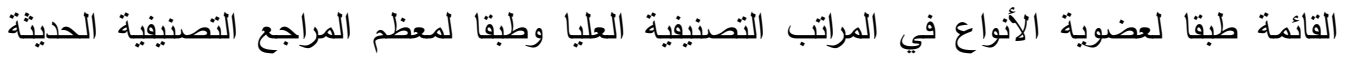
لفصيلة هاليكتيدى. 